\title{
NIVELES DE ANSIEDAD EN LA CONSULTA ODONTOLÓGICA EN PACIENTES ADULTOS ATENDIDOS EN LA UNIVERSIDAD SANTO TOMÁS
}

\author{
${ }^{1}$ Carmen Alodia Martínez López, ${ }^{2}$ Angie Paola Ramos Castañeda, ${ }^{2}$ María Alejandra Mantilla García, ${ }^{2}$ Alejandra Lizeth Duran Rueda, \\ ${ }^{3}$ Leslie Andreina Valencia Rodríguez

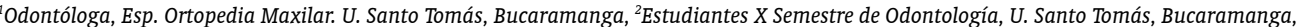 \\ ${ }^{3}$ Laboratorista dental, estudiante X Semestre de Odontología, U. Santo Tomás, Bucaramanga
}

\begin{abstract}
Autor responsable de correspondencia: Carmen Alodia Martínez López
Correo electrónico: carmen.martinez@ustabuca.edu.co

\section{RESUMEN}

Objetivos: determinar los niveles de ansiedad en pacientes adultos que asisten a consulta y tratamiento odontológico, identificando presencia y frecuencia de factores desencadenantes de ansiedad y relacionarlos con las características sociodemográficas de la población de estudio.

Materiales y métodos: se realizó un estudio observacional analítico de corte trasversal, con una muestra de 355 pacientes adultos, atendidos en las Clínicas Odontológicas de la Universidad Santo Tomás de Floridablanca, en el primer semestre de 2014. Se aplicó una encuesta autodiligenciada, previamente validada, para establecer las características sociodemográficas de los participantes, como también el test de ansiedad dental de Norman Corah. La información se registró en una base de datos, utilizando el programa Epi-Info versión 3.2.2. Se manejó estadística descriptiva para la presentación de variables cualitativas, medidas de tendencia central y de dispersión para las variables cuantitativas. El análisis bivariado se realizó mediante la prueba de $\mathrm{Chi}^{2}$. Cada paciente aceptó voluntariamente participar mediante la firma del consentimiento informado.

Resultados: en 197 (55.6\%) de los participantes no se evidenció ansiedad; el nivel de ansiedad más frecuente observado fue moderado, para 102 participantes $(28,8 \%)$, y el nivel de ansiedad severo se presentó en 34 casos $(9,6 \%)$. Los procedimientos odontológicos que generaron mayores niveles de ansiedad en los pacientes atendidos fueron la endodoncia y la exodoncia. Se encontró asociación entre los diferentes niveles de ansiedad y las variables edad $(p=0.001)$, estado civil $(p=0,010)$ y nivel educativo $(p=0,036)$.

Conclusiones: la aplicación del test de Norman Corah permitió establecer que un alto porcentaje de pacientes no refiere ansiedad frente a la atención odontológica, y en los pacientes que la reportan la ansiedad moderada es la más representativa. Los tratamientos de endodoncia y exodoncia requieren de anestesia troncular o infiltrativa, lo cual se encontró que aumenta la ansiedad durante el procedimiento. [Martínez CA, Ramos AP, Mantilla MA, Durán AL, Valencia LA. Niveles de ansiedad en la consulta odontológica en pacientes adultos de la Universidad Santo Tomás. Ustasalud. 2014;13(2): 112 - 119]
\end{abstract}

Palabras clave: Ansiedad dental, Consulta odontológica.

\section{ANXIETY LEVELS IN DENTAL CONSULTATION OF ADULT PATIENTS ATTENDED AT UNIVERSIDAD SANTO TOMÁS}

\begin{abstract}
Objectives: to determine the levels of anxiety in adult patients attending consultation and dental treatment, identifying the presence and frequency of triggers anxiety and relate them to the sociodemographic characteristics of the study population.

Methods: a cross- sectional study, with a sample of 355 adult patients attending dental clinics St. Thomas University in the first half of 2014. A survey previously validated was applied to establish the sociodemographic characteristics of the participants, as well as the test of dental anxiety of Norman Corah.

The information was recorded in a database using the Epi -Info version 3.2.2 program, descriptive statistics for qualitative variables measures of central tendency and dispersion for quantitative variables was used, for bivariate analysis Chi squared test was used. Each patient voluntarily agree to participate.

Results: there was no evidence of anxiety in 197 (55.6\%) subjects; the most frequently anxiety level was "moderate" for 102 participants $(28.8 \%)$, and "severe anxiety" in 34 cases $(9.6 \%)$. The dental procedures that generated higher levels of anxiety were endodontic and dental extraction. We found an association between the different levels of anxiety and the variables age $(p=0.001)$, marital status ( $p$ $=0.010)$ and educational level $(\mathrm{p}=0.036)$.

Conclusions: the application of the test of Norman Corah allows establish that a high percentage of patients referred no anxiety about dental care and patients who reported moderate anxiety is the most representative. Endodontic treatments and extraction require troncular or infiltrative anesthesia, which increases anxiety found during the procedure.
\end{abstract}

Key words: Dental anxiety, Dental health services.

Recibido para publicación: 13 de noviembre de 2014. Aprobado para publicación: 18 de diciembre de 2014 . 


\section{INTRODUCCIÓN}

La ansiedad se define como una sensación desapacible, la cual se anticipa a un acontecimiento desagradable o indeseable que está a punto de suceder. Está acompañada de síntomas como tensión muscular, estados de agitación y angustia. Dicha sensación está asociada a la reacción que tiene el individuo hacia un peligro desconocido $0^{1,2,3}$.

Cuando se habla de ansiedad es normal para diversos autores confundir este término con angustia, miedo o estrés, por lo cual en muchas ocasiones resulta dificil encontrar la definición adecuada para evidenciar realmente lo que es la ansiedad, sin embargo, dichos términos tienden a relacionarse lo suficiente para poder decir que la ansiedad puede llevar consigo altos niveles de miedo, estrés y hasta la angustia misma ${ }^{1,3,4}$.

El trastorno se presenta en ocasiones en personas con historia de experiencias traumáticas, lo que las hace vulnerables, aumentando sus temores. Cuando hay ansiedad, el individuo evita cualquier tipo de situación que tenga relación con la experiencia negativa ${ }^{5}$. Las actitudes de evasión y angustia a las situaciones que generan ansiedad afectan directamente la normalidad de las actividades laborales, académicas o sociales de las personas. Según el libro Fobias específicas de Arturo Bados López, las personas que padecen ansiedad están estrechamente relacionadas con la presencia de fobias ${ }^{6}$.

La clasificación de ansiedad dental de Norman Corah implementada en este trabajo es realizada por niveles. Cada nivel presenta manifestaciones diferentes que varían en intensidad y tienen una incidencia sobre la respuesta de la persona, en sus diferentes dimensiones (fisiológica, cognitiva y corporal). Originalmente se basa en cuatro preguntas con cinco opciones de respuesta y valores de 1 a 5 para casa opción. La puntuación máxima es de 20 y a partir de 15 el paciente es considerado con ansiedad severa o fobia. El mismo autor creó un cuestionario de preocupaciones dentales que ayuda a determinar cuáles son los factores desencadenantes de ansiedad en la consulta odontológica. Cabe aclarar que muchos autores se refieren al test como confiable y fácil de aplicar sobre todo en pacientes adultos ${ }^{4,7-9}$.

Ríos y colaboradores confirman las definiciones anteriormente descritas al considerar la ansiedad como la sensación de tipo subjetivo, experimentada por el individuo ante una situación que considera puede llegar a ser molesta en alto grado ${ }^{10}$. A partir del sentimiento de ansiedad el sujeto puede enfrentarse a la situación o huir de ella como mecanismo de defensa. Desde este punto de vista Ríos y colaboradores y Rojas y colaboradores, hacen énfasis en que la asistencia a odontología genera ansiedad, por cuanto el paciente no posee control de la situación y en ocasiones no hay predictibilidad favorable sobre el procedimiento. ${ }^{10,11}$

Se afirma que la ansiedad dental llega a ser más común que en otras situaciones de atención en sa$\operatorname{lud}^{12}$. Existen estudios que afirman que entre el 5 y 20 por ciento de las inasistencias a la consulta odontológica obedecen a altos niveles de ansiedad experimentados por los pacientes ${ }^{13,14}$. Posiblemente la relación directa que tienen los tratamientos odontológicos con dolor localizado, las experiencias traumáticas propias o provenientes de familiares o amigos hace que la odontología genere el sentimiento de ansiedad y sea razón suficiente para la interrupción de los tratamientos ${ }^{15}$.

La presencia de ansiedad también hace susceptible al paciente a presentar una disminución del umbral del dolor, convirtiéndose en un círculo vicioso, ya que el paciente no va a consulta odontológica por temor y cuando asiste efectivamente tiende a presentar mayor intensidad de dolor ${ }^{16-19}$. El profesional al percibir la situación del paciente puede contagiarse de la misma sensación de ansiedad, en ocasiones generando un trato inadecuado e insatisfactorio hacia el paciente. La relación negativa que se crea provoca desánimo de las dos partes y resulta fácil abortar el tratamiento ${ }^{20,21,22,23}$. Algunas estrategias son favorables para disminuir los niveles de ansiedad en los pacientes. Se requiere el conocimiento previo de la población, sus características y factores asociados para tratar la ansiedad y crear distractores que faciliten la atención y mejoren la relación paciente odontólogo ${ }^{8}$.

\section{MATERIALES Y MÉTODOS}

Se realizó un estudio observacional analítico de tipo transversal, cuyo objetivo fue determinar los factores asociados a la ansiedad dental. Se obtuvo una muestra no probabilística por conveniencia de 355 pacientes adultos, con edades entre 18 y 80 años, que asistieron a las Clínicas Odontológicas de la Universidad Santo Tomás Campus Floridablanca, durante el primer semestre de 2014.

Se aplicó a todos los participantes una encuesta previamente validada, para establecer las características sociodemográficas de interés (edad, género, estrato socioeconómico, nivel educativo, estado civil). Se empleó el test de ansiedad dental de Norman Corah, los resultados del instrumento se evaluaron en una escala de 1 a 20 puntos, de acuerdo con la respuesta señalada por el encues- 
tado $(a=1, b=2, c=3, d=4, e=5)$. La suma de estos valores se clasificó de la siguiente manera: (0-8 $=$ no ansiedad), $(9-12=$ ansiedad moderada) con factores de estrés específicos que deben ser discutidos y manejados; (13-14= ansiedad alta), en la cual el individuo posee dificultad para concentrarse y (15-20 = ansiedad severa) en la que hay percepción alejada de la realidad y que debe ser manejada por el odontólogo con apoyo farmacológico o psicológico ${ }^{4,7,9,10}$.

La aplicación de la encuesta sociodemográfica y el test de Norman se realizó por autodiligenciamiento. La información se registró en una base de datos, utilizando el programa Epi-Info versión 3.2.2. Se calculó la estadística descriptiva para las variables cualitativas, medidas de tendencia central y de dispersión para las variables cuantitativas. Se aplicó la prueba estadística de $\mathrm{Chi}^{2}(\mathrm{p}<0.05)$ para establecer la relación entre niveles de ansiedad y variables sociodemográficas de interés.

\section{Consideraciones Éticas}

Teniendo en cuenta la Resolución 008430 de 1993 del Ministerio de Salud, la presente investigación se clasificó según el artículo 11 como una investigación "sin riesgo". Se garantizaron los principios de beneficencia, equidad, confidencialidad y veracidad en el manejo de la información. El protocolo del trabajo recibió aprobación del Comité de Investigaciones de la Facultad de Odontología de la Universidad Santo Tomás. Todos los participantes otorgaron el consentimiento informado de manera verbal y escrito ${ }^{24}$.

\section{RESULTADOS}

La muestra estuvo conformada por 355 pacientes de los cuales el $47,3 \%$ (168) pertenece al sexo masculino con una edad promedio de $35,5 \pm$ (DS) 15,1 años y el 52,7\% (187) pertenece al sexo femenino, con una edad promedio de $38 \pm 14$ años.

El rango de edad entre los 18 y 28 años es de mayor frecuencia, con 134 adultos $(37,9 \%)$. El estrato socioeconómico 3 tuvo mayor representación con 136 pacientes (38,3\%); el nivel educativo predominante fue la secundaria, con 117 pacientes $(27,3 \%)$. En relación con el estado civil, las personas solteras asistieron a la consulta con mayor frecuencia: $145(40,8 \%)$. La medición de niveles de ansiedad permitió determinar que la mayoría de los pacientes entrevistados: $197(55,6 \%)$ no presentaron nivel alguno de ansiedad; con nivel de ansiedad moderado se identificaron 102 pacientes $(28,8 \%)$ y 34 pacientes $(9,6 \%)$ fueron detectados con ansiedad severa (Tabla 1).
Tabla 1. Descripción de características sociodemográficas y niveles de ansiedad de pacientes que asistieron a la Clínica Integral del Adulto I

Frecuencia Porcentaje (\%)

\section{Edad categorizada}

$\begin{array}{lcc}18 \text { а } 28 & 134 & 37,9 \\ 29 \text { а } 39 & 90 & 25,4 \\ 40 \text { а } 50 & 58 & 16,4 \\ 51 \text { а } 61 & 46 & 13 \\ 62 \text { а } 72 & 20 & 5,6 \\ 73 \text { а } 83 & 6 & 1,7\end{array}$

$\begin{array}{lll}\text { Masculino } & 168 & 47,3 \\ \text { Femenino } & 187 & 52,7\end{array}$

\section{Estrato social}

\begin{tabular}{lcc} 
Estrato 1 & 26 & 7,3 \\
Estrato 2 & 69 & 19,4 \\
Estrato 3 & 136 & 38,3 \\
Estrato 4 & 90 & 25,4 \\
Estrato 5 & 29 & 8,2 \\
Estrato 6 & 5 & 1,4 \\
\hline
\end{tabular}

Nivel educativo

\begin{tabular}{lcc} 
Primaria & 65 & 18,3 \\
Secundaria & 117 & 33,0 \\
Universitario & 97 & 27,3 \\
Técnico & 73 & 20,6 \\
Otro & 3 & 0,8 \\
\hline
\end{tabular}

Estado civil

\begin{tabular}{lcc} 
Soltero & 145 & 40,8 \\
Casado & 120 & 33,8 \\
Unión libre & 51 & 14,4 \\
Divorciado & 18 & 5,1 \\
Viudo & 21 & 5,9 \\
\hline
\end{tabular}

Niveles de ansiedad

$\begin{array}{lcc}\text { Sin Ansiedad } & 197 & 55,6 \\ \text { Ansiedad moderada } & 102 & 28,8 \\ \text { Ansiedad alta } & 21 & 5,9 \\ \text { Ansiedad severa } & 34 & 9,6\end{array}$


En cuanto al test de preocupaciones dentales se encontró que presentaron un nivel alto de ansiedad: $147(41,5 \%)$ pacientes a la extracción dental; 124 $(35,0 \%)$ pacientes al tratamiento de endodoncia y $117(33,1 \%)$ pacientes a los procedimientos de anestesia. El sonido o la vibración del motor fue el factor que más se asoció con la ansiedad, 108 (30,4\%) pacientes con nivel bajo, y con nivel medio 104 $(29,3 \%)$. La insuficiente información acerca de los procedimientos generó un bajo nivel de ansiedad en $102(28,7 \%)$ de los pacientes. Por último, el procedimiento que con mayor frecuencia fue considerado como un factor para no presentar ansiedad, consistió en la toma de radiografias (Tabla 2).

$\mathrm{Al}$ relacionar los niveles de ansiedad con los rangos de edad, se encontró que hay diferencias estadísticamente significativas entre los diferentes grupos de edad y presentar algún nivel de ansiedad. Los pacientes con edades entre 29 y 39 años son los que más tienden a referir algún nivel de ansiedad. No se encontró relación significativa entre las variables sexo y estrato con los niveles de ansiedad. El análisis estadístico sugiere que existe relación entre los niveles de ansiedad y el estado civil, ya que los pacientes con estado civil, casado, tuvieron mayor frecuencia de presentar algún nivel de ansiedad. De la misma manera, se encontró relación en la variable nivel educativo secundario con mayor frecuencia a presentar algún nivel de ansiedad (Tabla 3).

\section{DISCUSIÓN}

La presencia de ansiedad dental no deja de ser un tema de actualidad por cuanto genera expectativas negativas al paciente y al profesional, por lo que ella se relaciona con renuencia a recibir tratamiento aun cuando sea necesario ${ }^{10,16}$. Corah elaboró y sustentó por medio de estudios el test que permite determinar los niveles de ansiedad de los pacientes en diferentes grupos ${ }^{25}$. Se logró realizar este estudio considerando importante reconocer los estados de ansiedad que los pacientes pueden presentar en la consulta, debido a que niveles altos y severos deben ser atendidos de forma especial por el profesional, pues ellos generan deterioro de la salud dental, mayor necesidad de tratamiento, baja armonía en la relación odontólogo-paciente y en consecuencias el costo y molestias de tratamiento serán cada vez mayores ${ }^{9,11}$.

Los reportes de ansiedad de investigadores de otros países permiten observar que hay ansiedad alta hacia la consulta odontológica hasta en el 35\% de niños y el $41 \%$ de adultos en Sudamérica. España reporta $45 \%$ de no ansiedad, $36 \%$ ansiedad moderada y $19 \%$ ansiedad alta $^{17}$; otros países europeos hablan de presencia de ansiedad desde el $4 \%$ hasta el $36 \%$ de la población. En Estados Unidos se han obtenido datos de ansiedad severa en el $4 \%$ de la población y ansiedad moderada en un $11 \% \%^{19,21,22,26}$. El presente estudio establece diferencias en cuanto los niveles de ansiedad, siendo la ansiedad severa registrada en el $9.6 \%$ de la población, ansiedad alta en 5,9\% y moderada en $28.8 \%$. Estos datos llevan a pensar que el nivel de desarrollo de los países tiene influencia significativa en la aceptación del tratamiento odontológico, siendo más negativa la respuesta frente a la consulta en los países con menor desarrollo técnico y económico.

Llama la atención que en Bogotá, Caycedo y colaboradores en el 2008 comentan que al aplicar dos escalas de medición de ansiedad se obtuvo ansiedad severa en 25,6\% para STAI (State Trait Anxiety Inventory) y 27,4\% para MDAS (Modified Dental Anxiety Scale) ${ }^{4}$. Podría pensarse que ciudades donde se maneja nivel estrés alto debido a los tiempos de desplazamiento, aglomeraciones e inseguridad ${ }^{27}$, este suma al momento de evaluar la ansiedad en la consulta dental.

Los datos reportados por Caycedo son similares a los de Firat y colaboradores ${ }^{28}$ en el 2006, con $21 \%$ de ansiedad severa en población de Turquía donde las condiciones de la población son diferentes, pues el mayor porcentaje es de jóvenes menores de 28 años y el número de mujeres es bajo debido al comportamiento feudal de los habitantes ${ }^{29}$. Los datos revelados podrían deberse entonces a que la ansiedad es transmitida por terceros, por experiencias traumáticas previas, y tendrían validez desde la afirmación de que el aspecto cognitivo es un factor relevante en el desarrollo de la ansiedad ${ }^{7,10,30}$.

En lo que se refiere a la ansiedad moderada, en el presente estudio resultó ser de $28 \%$, coincidiendo cercanamente con estudios como los de Ríos ${ }^{10,17}$ y Arrie$\mathrm{ta}^{31}$, este último reporta valores de $36 \%$ para ansiedad leve y $21 \%$ para moderada, según test de Norma Corah modificado, en población adulta que asiste a la Facultad de Odontología de la Universidad de Cartagena. Los datos cercanos dejan ver que las características de los pacientes adultos son coincidentes en cuanto a las sensaciones percibidas ante la consulta odontológica donde los posibles factores ambientales relacionados con el entorno psicosocial y factores específicos como la naturaleza propia del individuo contribuyen a desencadenar estados de ansiedad.

La ansiedad moderada hallada en el presente estudio difiere significativamente con un estudio realizado en Jordania en el que el $91 \%{ }^{14}$ de la muestra presentó ansiedad moderada, un dato que deja la ansiedad severa y alta en mínimos porcentajes y que define a los pacientes como de manejo factible 
en la consulta. El hecho de que en el 2014 Jordania fue catalogada como un país con alto desarrollo humano ${ }^{32}$, permite pensar que se constituye en un factor ambiental que influye positivamente en la salud disminuyendo los niveles de ansiedad de la población.

Tabla 2. Factores desencadenantes de ansiedad (Cuestionario Dental de Norman Corah) aplicado a pacientes de la Clínica Integral del Adulto I, 2014

\begin{tabular}{|c|c|c|c|c|}
\hline & BAJO & MEDIO & ALTO & NO \\
\hline PROCEDIMIENTO & $\mathrm{n}(\%)$ & $\mathrm{n}(\%)$ & $\mathrm{n}(\%)$ & n ( \%) \\
\hline Sonido o vibración del motor & $108(30,4)$ & $104(29,3)$ & $67(18,9)$ & $76(21,4)$ \\
\hline Estar lo suficientemente tensionado en el procedimiento & $88(24,8)$ & $99(27,9)$ & $32(9)$ & $136(38,3)$ \\
\hline Miedo a estar tensionado & $89(25,1)$ & $92(25,9)$ & $35(9,9)$ & $139(39,2)$ \\
\hline Anestesia & $79(22,3)$ & $94(26,6)$ & $117(33,1)$ & $64(18,1)$ \\
\hline Sondaje para evaluar las enfermedades de las encías & $85(23,9)$ & $87(24,5)$ & $46(13)$ & $137(38,6)$ \\
\hline El sonido o la sensación de raspado durante la limpieza dental & $83(23,4)$ & $80(22,5)$ & $50(14,1)$ & $142(40)$ \\
\hline Nauseas, por ejemplo durante las impresiones dentales & $79(22,3)$ & $60(16,9)$ & $27(7,6)$ & $188(53,1)$ \\
\hline Toma de radiografias & $80(22,5)$ & $40(11,3)$ & $23(6,5)$ & $212(59,7)$ \\
\hline Tela de caucho para aislar dientes & $77(21,7)$ & $66(18,6)$ & $30(8,5)$ & $182(51,3)$ \\
\hline Cansancio mandibular & $81(22,8)$ & $71(20)$ & $37(10,4)$ & $166(46,8)$ \\
\hline Dolor dental al aplicar aire & $96(27)$ & $62(17,5)$ & $39(11)$ & $158(44,5)$ \\
\hline Insuficiente información acerca de los procedimientos & $102(28,7)$ & $71(20)$ & $40(11,3)$ & $142(40)$ \\
\hline Tratamiento de endodoncia (conductos) & $67(18,9)$ & $86(24,3)$ & $124(35)$ & $77(21,8)$ \\
\hline Extracción dental & $61(17,2)$ & $79(22,3)$ & $157(41,5)$ & $58(16,3)$ \\
\hline Miedo a ser herido & $97(27,3)$ & $67(18,9)$ & $91(25,6)$ & $100(28,2)$ \\
\hline Presentar ataques de pánico & $75(21,1)$ & $48(13,5)$ & $37(10,4)$ & $195(54,9)$ \\
\hline No poder detener al dentista & $83(23,4)$ & $54(14,4)$ & $35(9,9)$ & $185(52,3)$ \\
\hline No sentirse libre de hacer preguntas & $83(23,4)$ & $54(15,2)$ & $25(7)$ & $193(54,4)$ \\
\hline No ser escuchado o tomado en serio & $92(25,9)$ & $58(16,3)$ & $20(5,6)$ & $185(52,1)$ \\
\hline Ser criticado o regañado & $82(23,1)$ & $62(17,5)$ & $29(8,2)$ & $182(51,3)$ \\
\hline Olores en el consultorio dental & $95(26,8)$ & $48(13,5)$ & $22(6,2)$ & $190(53,5)$ \\
\hline Me preocupa que pueda necesitar muchos tratamientos dentales & $85(24)$ & $77(21,8)$ & $40(11,3)$ & $152(42,9)$ \\
\hline Estoy preocupado por el costo del tratamiento dental que necesite & $85(23,9)$ & $96(27)$ & $56(15,8)$ & $118(33,2)$ \\
\hline $\begin{array}{l}\text { Estoy preocupado por el número de citas y el tiempo que se } \\
\text { requerirá para los tratamientos necesarios, el tiempo fuera del } \\
\text { trabajo, la necesidad de cuidado de los niños o el transporte }\end{array}$ & $90(25,4)$ & $93(26,2)$ & $34(9,6)$ & $138(38,9)$ \\
\hline Estoy avergonzado por la condición de mi boca & $72(20,3)$ & $53(14,9)$ & $38(10,7)$ & $192(54,1)$ \\
\hline No me gusta no tener el control & $64(18)$ & $36(10,1)$ & $20(5,6)$ & $235(66,2)$ \\
\hline
\end{tabular}


Tabla 3. Descripción de los niveles de ansiedad según variables demográficas aplicado a pacientes de la Clínica Integral del Adulto I, 2014

\begin{tabular}{|c|c|c|c|c|c|c|}
\hline Variables & $\begin{array}{l}\text { Global } \\
\text { n (\%) }\end{array}$ & $\begin{array}{c}\text { Sin Ansiedad } \\
\mathbf{n}(\%)\end{array}$ & $\begin{array}{c}\text { Ansiedad } \\
\text { moderada } \\
\mathbf{n}(\%)\end{array}$ & $\begin{array}{c}\text { Ansiedad alta } \\
\mathbf{n}(\%)\end{array}$ & $\begin{array}{c}\text { Ansiedad } \\
\text { severa } \\
\text { n (\%) }\end{array}$ & Valor de $\mathbf{P}$ \\
\hline Edad & & & & & & 0,001 \\
\hline 18 a 28 & $134(100)$ & $93(26,3)$ & $31(8,8)$ & $5(1,4)$ & $5(1,4)$ & \\
\hline 29 a 39 & $90(100)$ & $39(11,0)$ & $27(7,6)$ & $7(2,0)$ & $17(4,8)$ & \\
\hline 40 a 50 & $58(100)$ & $31(8,8)$ & $22(6,2)$ & $1(3)$ & $4(1,1)$ & \\
\hline 51 a 61 & $46(100)$ & $20(5,6)$ & $17(4,8)$ & $3(8)$ & $6(1,7)$ & \\
\hline 62 a 72 & $20(100)$ & $12(3,4)$ & $3(0,8)$ & $3(0,8)$ & $2(0,6)$ & \\
\hline 73 a 83 & $6(100)$ & $2(0,6)$ & $2(0,6)$ & $2(0,6)$ & $0(0)$ & \\
\hline Género & & & & & & 0,297 \\
\hline Femenino & $187(100)$ & $97(51,9)$ & $55(29,4)$ & $13(7,0)$ & $22(11,8)$ & \\
\hline Masculino & $167(100)$ & $100(59,9)$ & $47(28,1)$ & $8(4,8)$ & $12(7,2)$ & \\
\hline Estrato & & & & & & 0,340 \\
\hline Estrato 1 & $26(100)$ & $12(46,2)$ & $9(34,6)$ & $0(0)$ & $5(19,2)$ & \\
\hline Estrato 2 & $69(100)$ & $32(46,4)$ & $23(33,3)$ & $4(5,8)$ & $10(14,5)$ & \\
\hline Estrato 3 & $136(100)$ & $78(57,4)$ & $34(25)$ & $12(8,8)$ & $12(8,8)$ & \\
\hline Estrato 4 & $89(100)$ & $53(58,9)$ & $28(31,1)$ & $3(3,3)$ & $5(5,6)$ & \\
\hline Estrato 5 & $29(100)$ & $19(65,5)$ & $7(24,1)$ & $2(6,9)$ & $1(3,4)$ & \\
\hline Estrato 6 & $5(100)$ & $3(60)$ & $1(20)$ & $0(0)$ & $1(20)$ & \\
\hline Estado civil & & & & & & 0,010 \\
\hline Soltero & $145(100)$ & $96(66,2)$ & $32(22,1)$ & $7(4,8)$ & $10(6,9)$ & \\
\hline Casado & $119(100)$ & $61(51,3)$ & $41(34,5)$ & $7(5,9)$ & $10(8,4)$ & \\
\hline Unión libre & $51(100)$ & $21(41,2)$ & $19(37,3)$ & $2(3,9)$ & $9(17,6)$ & \\
\hline Divorciado & $18(100)$ & $9(50,0)$ & $7(38,9)$ & $1(5,6)$ & $1(5,6)$ & \\
\hline Viudo & $21(100)$ & $10(47,7)$ & $3(14,3)$ & $4(19,0)$ & $4(19,0)$ & \\
\hline Nivel educativo & & & & & & 0,036 \\
\hline Primaria & $65(100)$ & $29(8,2)$ & $19(5,3)$ & $6(1,7)$ & $11(3,1)$ & \\
\hline Secundaria & $117(100)$ & $57(16,6)$ & $43(12,1)$ & $7(1,9)$ & $10(2,8)$ & \\
\hline Universitaria & $96(100)$ & $65(18,3)$ & $21(5,9)$ & $1(0,3)$ & $9(2,5)$ & \\
\hline Técnico & $73(100)$ & $44(12,3)$ & $18(5,1)$ & $7(2)$ & $4(1,1)$ & \\
\hline Otros & $3(100)$ & $2(0,6)$ & $1(0,3)$ & $0(0)$ & $0(0)$ & \\
\hline
\end{tabular}

*Prueba $\mathrm{Chi}^{2}$, $\mathrm{p}<0.05$

Dentro de la muestra de este estudio, el sexo femenino es más propenso a presentar estados de ansiedad frente a la consulta odontológica (con una diferencia que no fue significativa respecto a la ansiedad severa en comparación con el género masculino), la mayoría de estudios aquí citados señalan datos similares ${ }^{4,10,31}$, destacando un estudio hecho en Pakistán donde la diferencia de la muestra entre hombres y mujeres es bastante significativa ${ }^{20}$. La explicacion aceptada para muchos investigado- res acerca de mayor ansiedad en mujeres radica en patrones culturales, aceptación social hacia las mujeres para expresar dolor, problemas familiares y relación directa con situaciones hormonales ${ }^{33}$.

En cuanto a los factores desencadenantes un nivel de ansiedad alto estuvo relacionado con extracción dental $(41,5 \%)$, tratamiento endodóntico $(35,0 \%)$ y procedimiento de aplicación de anestesia $(33,1 \%)$. Caycedo reporta $47,5 \%$ de ansiedad ante la anestesia y $36,8 \%$ frente a la fresa dental. Por su parte 
Kleinknecht ${ }^{34}$ en su estudio relacionó porcentajes mayores al 30\% con ansiedad elevada en pacientes por el hecho de ver y sentir la aguja anestésica y la turbina de alta velocidad, y describió el resultado como una respuesta condicionada sin existencia de condición real para sentir ansiedad.

Skaret afirma que el miedo dental es adquirido dados los conceptos negativos hacia el profesional y los procedimientos en conversaciones en el seno de la familia ${ }^{30}$. Lo cierto al parecer es que los procedimientos odontológicos generan sensación de inseguridad por los instrumentos y ruidos, hace falta mayor instrucción y preparación del paciente y del odontólogo para aceptar terapias de relajación antes de enfrentar un tratamiento odontológico ${ }^{35}$.

Las limitaciones de este trabajo representadas en el diseño transversal, el muestreo realizado por conveniencia y la falta de representatividad de la muestra, hacen que las conclusiones de este trabajo deban interpretarse con precaución. Se sugiere explorar las hipótesis aquí generadas en estudios con un mayor tamaño de muestra y de tipo longitudinal.

\section{Agradecimientos}

Los autores desean expresar un especial agradecimiento a las personas que aceptaron participar en el estudio.

\section{BIBLIOGRAFÍA}

1. Sierra JC, Ortega V, Zubeidat I. Ansiedad, angustia y estrés: tres conceptos a diferenciar. Malestar e subjetividad. 2003 Mar 3(1):10-59;

2. [Online] 2013 May 63(5):614-618.[Fecha de acceso: 7-052014]. Disponible en: http://www. jpma.org.pk/ PdfDownload/4183.pdf

3. Hazem K, Ayman O. Dental Anxiety and its Possible Effects on Caries Prevalence. Journal of the royal medical services. 2013 Jun 20(2):26-31.

4. Caycedo M, Colorado P, Barahona G, Palencia R et al. Ansiedad al tratamiento odontológico: característi- cas y diferncias de género. Suma psicológica. $2008 \mathrm{Mar}$ 15(1):259-278.

5. Sana H, Manal A. Dental anxiety: prevalence and associated factors. European J Gen Dent. 2013 Sep 2(3):270273.

6. Ehud B, Lulian L. Recalling the Threat: dental anxiety in patients waiting for dental surgery. Isr J Psychiatry Relat Sci. 2013;50(1):61-67.

7. Corah NL, O'Shea R, Skeels DK: Dentists' perceptions of problem behaviors in patients. Journal of the American Dental Association. 1982;104(6):829-833.

8. Corah NL. Development of denal anxiety scale. Journal of Dental Reseaech. 1969;48(84):596.

9. Pérez P. Estudio de la ansiedad y el miedo dental en una muestra de universitarios de diferentes licenciaturas. Trabajo doctoral. Valencia: Universidad de Valencia; 2011.
10. Ríos M, Herrera A, Rojas G. Ansiedad dental: evaluación y tratamiento. Avances en odontoestomatología. 2014;30(1):39-46.

11. Rojas G, Harwarst P, Molina A, Molina Y, Herrera A, Ríos M, Misrachi C. Eficacia de las técnicas de percepción de control y relajación en la reducción de ansiedad dental. Acta Odontológica [Internet]. 2011[citado 2013 jun 25];49(4). Disponible en: http:/ www.actaodontologica. com/ediciones/2011/4/art-6/\#

12. Mercado $F$. The influence of emotional contexto attention in anxious subjects: neurophysiological correlates. Journal of Anxiety Disorder. 2006;20(1):72-84.

13. Berggren U. Meynert G. Dental fear and avoidance: causes symptoms and consequences. Journal of the American dental association, 1984;109(2):247-251.

14. Rowe M, Moore T. Self-Report Measures of Dental fear: Gender Differences. American Journal of Health Behaviour. 1998;22(4):243-247.

15. Valencia M, Bedoya LM, Vinaccia S. Odontología y psicología. Disminución de la ansiedad en pacientes someti- dos a cirugía odontológica. Revista Latinoamericana de Psicología [Internet]. 1998 [citado 2014 oct 13];30. Disponible en: <http://www.re-dalyc.org/articulo.oa? id $=80530104>$ ISSN 0120-0534

16. Fregozo C, Zonta E, Llanos H, Olivares R. La odontología, el dolor y la ansiedad. Revista Nacional de Odontología. 2009;1:12-13.

17. Díaz EM, San Martín LD, Ruiz LG, Veguilla M. Miedo al dentista e intervenciones para reducirlo. Anuario de psicología clínica y de la salud. 2013 ;9:53-55.

18. Attaullah, Ayyan AK. Prevalence of dental anxiety among university students in islamabad, pakistan. JKCD. 2011 Jun 1(2):71-77.

19. Katarzyna D, Justyna H, Anna W, Joanna Z, Dominik R. The level of dental anxiety and dental status in adult patients. Jl of Int Oral Health[Online]. 2014 Mar; 6(3):11-14.

20. Sghaireen M, Zwiri A, Alzoubi I, Qodceih S, Mahmoud K. Anxiety due to dental treatment and procedures among university students and its correlation with their gender and field of study. International journal of dentistry [Internet]. 2013 Feb [citado 2014 oct 16]. Disponible en: http://www.hindawi.com/journals/ijd/2013/647436/

21. Bernson JM, Elfstrom ML, Hakeberg M. Dental coping strategies, general anxiety, and depression among adult patients with dental anxiety but with different dentalattendance patterns. Eur J of Oral Sci. 2013;121:270-6.

22. Gaffar B, Alagl A, Al-Ansari A. The prevalence, causes, and relativity of dental anxiety in adult patients to irregular dental visits. Saudi Med J. 2014;35(6):598-603.

23. Krahn N, García A, Gómez L, Astié F. Fobia al tratamiento odontológico y su relación con ansiedad y depresión. Fundamentos en Humanidades. 2011;7(23):213-222.

24. Republica de Colombia. Ministerio de Salud. Resolución 008430. Artículo 11 (1993).

25. Corah NL, Gale E, Illig S. Assessment of a dental anxiety scale. J Am Dent Assoc. 1978;97:816-819.

26. Muza R, Muza P. Niveles de preocupacion o ansiedad sobre procedimientos dentales en una sala de espera dental. Revista chile salud publica. 2007;11(1):18-22.

27. Otálora G, La relación existente entre conflicto trabajofamilia y el estrés. Rev. Adm. 2007:20(34):139-160. 
28. Firat D, Tunc EP, Sar V. Dental anxiety among adults in Turkey. J Contemp Dent Pract 2006;3(7):75-82.

29. Dymond J. Turkish girls in literacy battle. British Broadcasting Corporation. BBC News. 2014 oct 18.

30. Skaret E, Raadal M, Berg E, Kvale G. Dental anxiety among 18 yr. olds in Norway. Prevalence and related factors. Eur J Oral Sci 1998;106(4):835-843.

31. Arrieta K, Díaz S, Cárdenas J. Bohórquezc V, Hawasly N. Factores asociados a sintomatología clínica de miedo y ansiedad en pacientes atendidos en Odontología. Rev Clín Med Fam. 2013; 6(1):17-24.

32. Programa de las Naciones Unidas para el Desarrollo (PNUD) [Internet]. "Informe sobre el desarrollo humano" 2014 [citado 2013 ago 24]. Disponible en: http://hdr.undp. org/sites/default/files/hdr14-summary- es.pdf Informe sobre Desarrollo Humano 2014

33. Álvarez ML, Casanova Y, Amador C, Espeso N. Mie- do al tratamiento estomatológico en pacientes de la clínica estomatológica docente provincial. Rev Hum Med [Internet] 2007 [citado 2013 jun 14];7 (1). Disponible en: http://scielo.sld.cu/scielo.php? pid=S1727$81202007000100006 \&$ script $=$ sci_arttext
34. Kleinknecht R, Klepac R, Alexander L.: Origins and characteristics of fear of dentistry. J Am Dent Assoc. 1973;86:842848.

35. Márquez-Rodríguez JA, Navarro-Liranzazu MC, Cruz-Rodríguez D, Gil-Flores J. ¿Por qué se le tiene miedo al dentista? Estudio descriptivo de la posición de los pacientes de la sanidad pública en relación a diferentes factores subyacentes a los miedos dentales. RCOE, 2004;9(2):165-174.

Correo electrónico de los autores:

Carmen Alodia Martínez López: carmen.martinez@ustabuca.edu.co Angie Paola Ramos Castañeda: apramosc@outlook.es

María Alejandra Mantilla García: alejandramantilla1591@hotmail.com Alejandra Lizeth Durán Rueda: alejandrad406@gmail.com Leslie Andreina Valencia Rodríguez: leslie.valencia@outlook.es

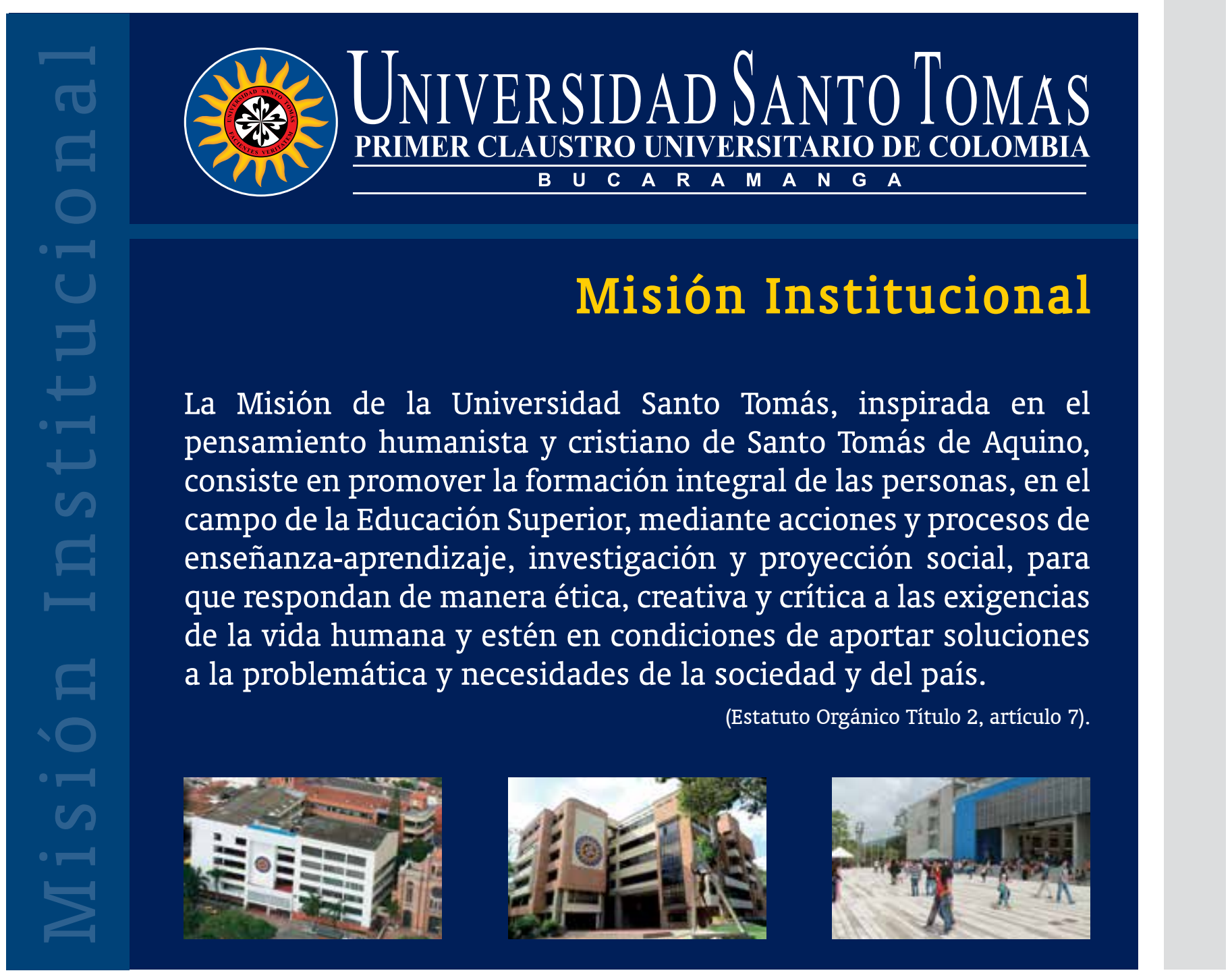

